\title{
Nail Fold
}

National Cancer Institute

\section{Source}

National Cancer Institute. Nail Fold. NCI Thesaurus. Code C34215.

Folds of epidermis surrounding the embryonic nail fields, with the proximal fold eventually keratinizing to form the nail plate. 